\title{
Haste Makes Waste: The Interplay Between Dissolution and Precipitation of Supersaturating Formulations
}

\author{
Dajun D. Sun ${ }^{1,2}$ and Ping I. Lee ${ }^{1,3}$
}

Received 9 June 2015; accepted 24 August 2015; published online 3 September 2015

\begin{abstract}
Contrary to the early philosophy of supersaturating formulation design for oral solid dosage forms, current evidence shows that an exceedingly high rate of supersaturation generation could result in a suboptimal in vitro dissolution profile and subsequently could reduce the in vivo oral bioavailability of amorphous solid dispersions. In this commentary, we outline recent research efforts on the specific effects of the rate and extent of supersaturation generation on the overall kinetic solubility profiles of supersaturating formulations. Additional insights into an appropriate definition of sink versus nonsink dissolution conditions and the solubility advantage of amorphous pharmaceuticals are also highlighted. The interplay between dissolution and precipitation kinetics should be carefully considered in designing a suitable supersaturating formulation to best improve the dissolution behavior and oral bioavailability of poorly water-soluble drugs.
\end{abstract}

KEY WORDS: amorphous formulation; kinetic solubility; nonsink dissolution testing; poorly watersoluble drug; supersaturation rate.

\section{INTRODUCTION}

Therapeutic candidates with poor aqueous solubility pose technical challenges in formulating oral dosage forms during pharmaceutical development. Modern solubilization technologies-with advances in lipid-based, selfmicroemulsifying, nano-sized, and amorphous formulations, among many others-have equipped formulation scientists with essential tools to develop poorly water-soluble compounds into viable drug products with adequate oral absorption. These enabling formulations can promote oral bioavailability by fundamentally increasing the drug's equilibrium solubility (e.g., prodrugs), enhancing the apparent solubility by forming drug-carrier complexes (e.g., surfactant micelles), or creating a supersaturated drug solution (e.g., amorphous solid dispersions (ASDs) in water-soluble carriers) during dissolution in the gastrointestinal (GI) microenvironment. In the latter case, precipitation within the intestinal lumen is a thermodynamically favored process owing to the unstable nature of the supersaturated drug solution which provides a driving force for nucleation and crystallization. If extensive supersaturation-induced precipitation occurs in the upper GI tract before the solubilized drug can be sufficiently absorbed, reduced absorption rate and suboptimal systemic exposure could occur. The rate and

\footnotetext{
${ }^{1}$ Department of Pharmaceutical Sciences, Leslie Dan Faculty of Pharmacy, University of Toronto, 144 College Street, Toronto, Ontario M5S 3M2, Canada.

${ }^{2}$ Food and Drug Administration, 10903 New Hampshire Avenue, Silver Spring, Maryland 20993, USA.

${ }^{3}$ To whom correspondence should be addressed. (e-mail: ping.lee@utoronto.ca)
}

extent of intestinal precipitation depends on many complex factors that directly affect the duration of drug supersaturation in the GI tract, such as the physicochemical properties of the drug molecules (e.g., pH-dependent solubility, ability to generate supersaturation, crystallization propensity), physiological factors (e.g., permeability across intestinal villi, absorption window, gastric emptying), food effect, excipient effect (e.g., crystallization inhibition), and formulation design (e.g., dissolution rate). During drug development, supersaturating formulations are often subjected to in vitro dissolution testing to gain a better understanding of their supersaturation kinetics and potential in vivo precipitation behavior. In this case, the resulting dissolution profiles under nonsink conditions have typically been characterized qualitatively by an initial rapidly dissolving and supersaturating "spring" with a precipitation retarding "parachute" $(1,2)$. In such a "spring-and-parachute" approach, the design rationales of supersaturating drug delivery systems have primarily been focused on enhancing the dissolution rate, increasing the maximum achievable supersaturation, and prolonging its duration following the dissolution by delaying the recrystallization of supersaturated drug solutions.

Historically, a consideration of the modified NoyesWhitney equation $(3,4)$ based on Fickian diffusion has provided scientific insights into how the dissolution kinetics of poorly water-soluble drugs can improve oral absorption:

$$
\frac{d m}{d t}=\frac{A D}{h}\left(C_{A}-C_{B}\right)
$$

where $m$ is the mass of dissolved drug, $t$ time, $A$ the surface area of the interface between the dissolving drug particle and the dissolution medium, and $D$ the diffusion coefficient of the 
drug. The term $\left(C_{A}-C_{B}\right) / h$ denotes the concentration gradient between the drug concentration on the particle surface $\left(C_{A}\right)$ and that in the bulk medium $\left(C_{B}\right)$ over the diffusion layer thickness, $h$, at the dissolving drug particle surface thus representing the driving force for drug release. The term $(d m / d t)$ here refers to the dissolution rate from a given surface area $(A)$ and is often estimated from the initial dissolution rate from spherical drug particles. Under sink dissolution conditions, the drug concentration in the bulk medium is considered negligible (i.e., $C_{\mathrm{B}}=0$ ); $D$ and $h$ are constants (note: $h$ is usually related to the agitation hydrodynamics). As a result, the dissolution rate is proportional to $A$ and $C_{A}$. According to this analysis, the dissolution rate can be enhanced by increasing the effective surface area available for dissolution (i.e., decreasing the particle size of the solids) or by improving the kinetic solubility of the drug under physiologically relevant conditions (i.e., employing ASDs). In early studies of amorphous pharmaceuticals, pioneering researchers such as Goldberg et al. $(5,6)$, Simonelli et al. $(7,8)$, and Chiou et al. (9) all conducted their dissolution experiments under sink conditions and thereby emphasized the importance of a high dissolution rate of amorphous drugs from solid dispersions (e.g., sulfathiazole-polyvinylpyrrolidone PVP, griseofulvinpolyethylene glycol PEG) or eutectic mixtures (e.g., acetaminophen-urea, griseofulvin-succinic acid) as an essential criterion for assessing the solubility improvement. Consequently, the concept of enhancing dissolution rates of amorphous pharmaceuticals by incorporating rapidly dissolving ASD carriers has been adopted and propagated to succeeding generations of formulation design for orally administered poorly water-soluble drugs. Solid dispersion technologies applied to commercial drug products have thus involved exclusively water-soluble carriers such as PVP (e.g., Cesamet $\left.{ }^{\circledR}\right)$, polyvinylpyrrolidone-vinyl acetate (PVP/VA) (e.g., Kaletra ${ }^{\circledR}$, Novir $\left.{ }^{\circledR}\right)$, hydroxypropyl methylcellulose (HPMC) (e.g., Sporanox $\AA$, Prograf $\AA)$, and hydroxypropyl methylcellulose acetate succinate (HPMCAS) (e.g., Zelboraf®, Kalydeco®) (10) to facilitate rapid dissolution in accordance with the early philosophy of formulation design. Dissolution enhancement using slowly dissolving or waterinsoluble matrices for creating the ASDs has gained relatively less attention despite their growing potential as a promising category of ASD carriers (11).

In a 2012 commentary, Augustijns and Brewster (12) described a conundrum concerning the "spring-and-parachute" design approach for supersaturating drug delivery systems, in that the higher the desired rate and extent of supersaturation, the more it exacerbates the physical instability of the metastable supersaturated drug solution due to an increased tendency for the solubilized drug to crystallize during dissolution. Specifically, the authors remarked on a general shortfall in correlating the in vitro and in vivo results: Fast dissolution of supersaturating formulations does not always produce better in vivo performance.

Here, we wish to highlight important recent experimental and theoretical evidence to help bridge the gap in our current understanding of the interplay between dissolution and precipitation kinetics of supersaturating drug delivery systems. As stated earlier, the in vivo pharmacokinetic performance of supersaturating formulations is a complex phenomenon which involves many processes that directly affect the duration of drug supersaturation in the GI tract. This commentary aims to address the in vitro in vivo implications of supersaturation generation rate using available literature in vivo data for supersaturating formulations. Through a better understanding of the critical effects of the supersaturation generation rate (i.e., dissolution rate) and the initial degree of supersaturation of amorphous pharmaceuticals on the resulting supersaturation kinetics, it is hoped that improvement of the design of enabling supersaturating formulations for oral drug delivery will emerge.

\section{IN VITRO IN VIVO RELATIONSHIP OF SUPERSATURATION GENERATION RATE}

Since insoluble crosslinked poly(2-hydroxyethyl methacrylate) (PHEMA) hydrogel has been shown to be an effective ASD carrier for immediate-release applications of several poorly water-soluble model drugs $(13,14)$, a head-to-head assessment of solubility enhancement on the kinetic solubility profiles of indomethacin ASD in PHEMA, PVP, and HPMCAS under nonsink dissolution conditions was conducted by Sun et al. (14) (note: key results are reproduced in Fig. 1). Here, the dissolution of indomethacin ASD from insoluble crosslinked PHEMA hydrogel beads was found to increase in a more gradual fashion before building up to a sustained supersaturation. This is most likely because drug release is governed by a matrix diffusion-regulated mechanism as compared with that generated from water-soluble ASD carriers such as PVP or HPMCAS, which exhibited a "spring-and-parachute" supersaturation kinetic profile governed by dissolution-controlled mechanism. As shown in Fig. 1 at a $32.9 \mathrm{wt} \%$ drug loading, the initial drug solubility improvement appears to be higher for ASDs based on watersoluble carriers (e.g., PVP and HPMCAS) but the extent of solubility improvement of indomethacin ASD in insoluble crosslinked PHEMA outperforms those in water-soluble carriers after $8 \mathrm{~h}$. In this case, the dissolution medium for the indomethacin-PHEMA ASD system remains supersaturated even after $24 \mathrm{~h}$ in the absence of any dissolved polymer acting as a crystallization inhibitor in the dissolution medium. In fact, other properly designed supersaturating formulations regulated by matrix diffusion (i.e., based on other waterinsoluble carriers) have recently been shown to exhibit similar advantageous kinetic solubility profiles where drug supersaturation is sustained in the absence of any crystallization inhibitor (15). Additional observations showing a more sustained supersaturation resulting from a more gradual drug release have also been reported for the dissolution of griseofulvin from synthetic hectorite (i.e., an insoluble swelling clay) (16) and nilvadipine from crosslinked PVP (17). These independent studies together provide strong supporting evidence that the rate of supersaturation generation plays an essential role in determining the level of transient solubility enhancement, thereby affecting the overall kinetic solubility profiles under nonsink dissolution conditions (note: the experimental and modeling verification of this effect of supersaturation generation rate will be discussed in the Section "DO NOT PUSH TOO FAR TOO FAST").

How do different supersaturation generation rates of amorphous pharmaceuticals affect the in vivo pharmacokinetic 

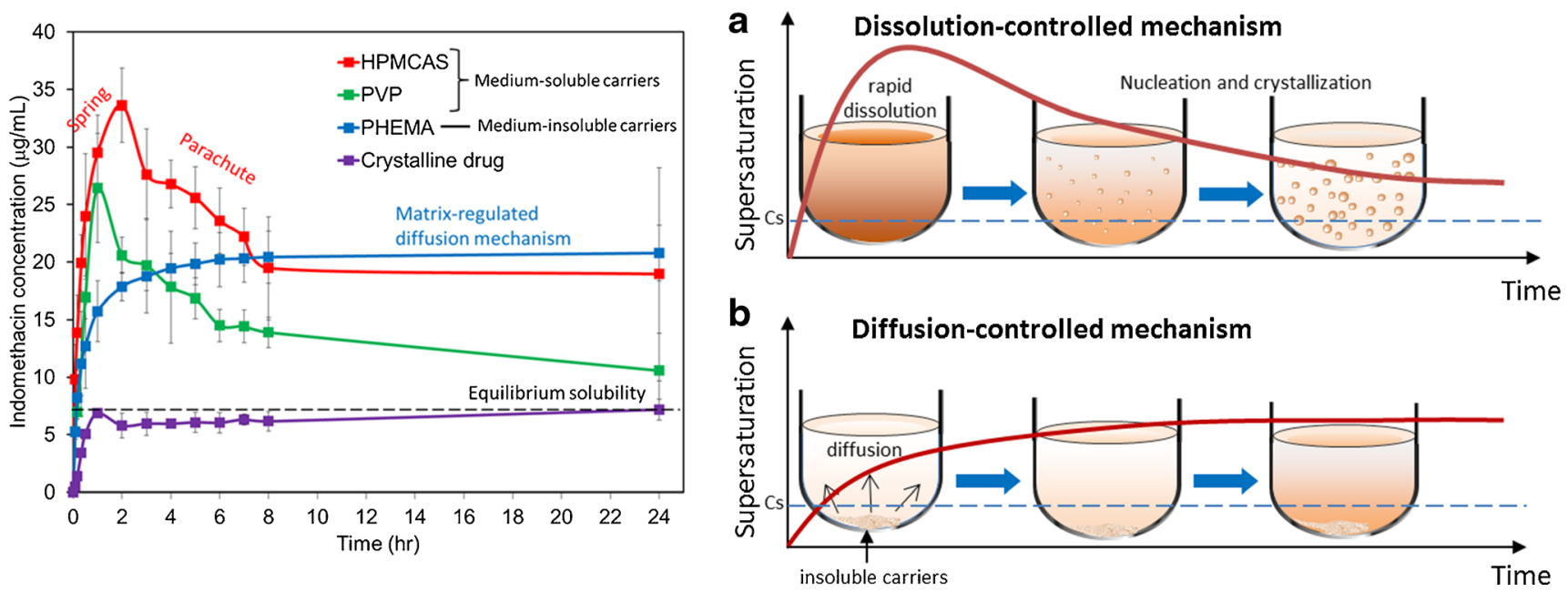

Fig. 1. Indomethacin release from amorphous solid dispersions at a drug loading of $32.9 \%$ in soluble carriers (PVP and HPMCAS) via a dissolution-controlled mechanism and insoluble carrier (PHEMA) via a diffusion-controlled mechanism under nonsink dissolution conditions $(\mathrm{SI}=0.1$ from Eq. 2). The dashed line represents the equilibrium solubility of indomethacin in the dissolution medium. Figures adapted in part from Sun et al. (14) and Sun and Lee (15) (reproduced with permission from the European Journal of Pharmaceutics and Biopharmaceutics and Journal of Controlled Release, Copyright Elsevier, 2012/2015)

performance? A clear evidence can be gleaned from the data of Van Speybroeck et al. who investigated the in vitro dissolution and in vivo oral absorption in rats of amorphous fenofibrate released from water-insoluble mesoporous silica formulations of different pore sizes which dictate the rates of drug release $(18,19)$. The dissolution profiles of fenofibrate from mesoporous silica show the highest dissolution rate from silica material with the largest pore size $(7.3 \mathrm{~nm})$ under sink dissolution conditions (Fig. 2a). When the same formulations are tested in dissolution experiments under different nonsink conditions (Fig. 2b, c), the fastest dissolution rate under the most nonsink conditions (characterized by the lowest SI value) results in an early onset of crystallization and a rapid de-supersaturation phase in the kinetic solubility profiles as shown in Fig. 2c. When the corresponding formulations were tested in vivo, the extent of oral bioavailability in rats under fasted conditions (Fig. 3) appears to be inversely related to the initial drug release rate while directly related to the area-underthe-curve (AUC) of the in vitro kinetic solubility concentrationtime profile shown in Fig. 2c. In contrast, the resulting pharmacokinetic profiles show more similar systemic exposure for all formulations under fed conditions (data not shown; see Fig. 6 of Ref. 18). The authors attributed this observed difference in absorption to the solubilization effect by food components as well as to an elevated equilibrium solubility of fenofibrate in fed-state simulated intestinal fluid (FeSSIF) $(53.8 \mu \mathrm{g} / \mathrm{mL})$ as compared to that in fasted-state simulated intestinal fluid (FaSSIF) $(13.6 \mu \mathrm{g} / \mathrm{mL})(18)$. This renders the in vivo absorption behavior under fed and fasted conditions comparable to the trends of AUCs in the corresponding nonsink in vitro dissolution profiles of Fig. 2b, c, respectively; the distinction between the nonsink conditions in these figures will be addressed in the next section.

Furthermore, a similar trend of the effect of supersaturation generation rate was also observed in water-soluble ASD systems. The pharmacokinetics of itraconazole in human volunteers after oral administration of single-dose Sporanox (i.e., a commercially available ASD formulation of itraconazole in HPMC) and other ASD systems prepared in Eudragit E100 and Eudragit E100-PVPVA64 has been investigated by Six et al. (20). Their results (Fig. 4) show that Sporanox and ASD in HPMC extrudate release itraconazole more slowly in in vitro dissolution testing under nonsink conditions (Sink Index $=0.02$ ) compared to other ASD systems (i.e., in Eudragit E100 and Eudragit E100-PVPVA64) (20). On the other hand, itraconazole ASD systems based on HPMC (including Sporanox) provided higher systemic exposure (i.e., a higher Cmax and AUC) (see Fig. 5). The mean oral bioavailability $(\mathrm{F})$ of itraconazole hot-melt extrudates based on HPMC, Eudragit E100, and Eudragit E100PVPVA64 in reference to commercially available Sporanox is $102.9,77.0$, and $68.1 \%$, respectively, with only the difference in mean AUC between Eudragit E100-PVPVA64 and Sporanox statistically significant but no difference between the other formulations as reported in the original paper (20). Thus, the mean bioavailability of the HPMC formulation is the same as that for Sporanox and the mean relative bioavailability is the lowest for the Eudragit E100PVPVA64 formulation. Similar to the in vivo in vitro trend observed in rats for the fenofibrate-silica ASD system based on water-insoluble carriers (Figs. 2 and 3), the extent of oral bioavailability in human subjects for the itraconazole ASD system based on water-soluble carriers also appears to be inversely related to the initial drug release rate (Figs. 4 and 5). However, no conclusion can be drawn here on relationship with the area-under-the-curve (AUC) of the in vitro kinetic solubility concentration-time profile as with the rat study because of the lack of such dissolution data at lower SI values. Here, the supersaturation generation rate plays an important role in the microenvironment in which the drug supersaturation can be adequately maintained for the slower releasing itraconazole ASD systems based on water-soluble carriers (i.e., Sporanox and ASD in HPMC extrudate). In addition, Six et al. also show that a modest dissolution rate of itraconazole from a solid dispersion achieved by a combination of fast and slowly releasing polymers reaches a sustained supersaturation without precipitation, whereas the dissolution of amorphous itraconazole released from the fast dissolving polymer causes precipitation (21). Based on these presented 

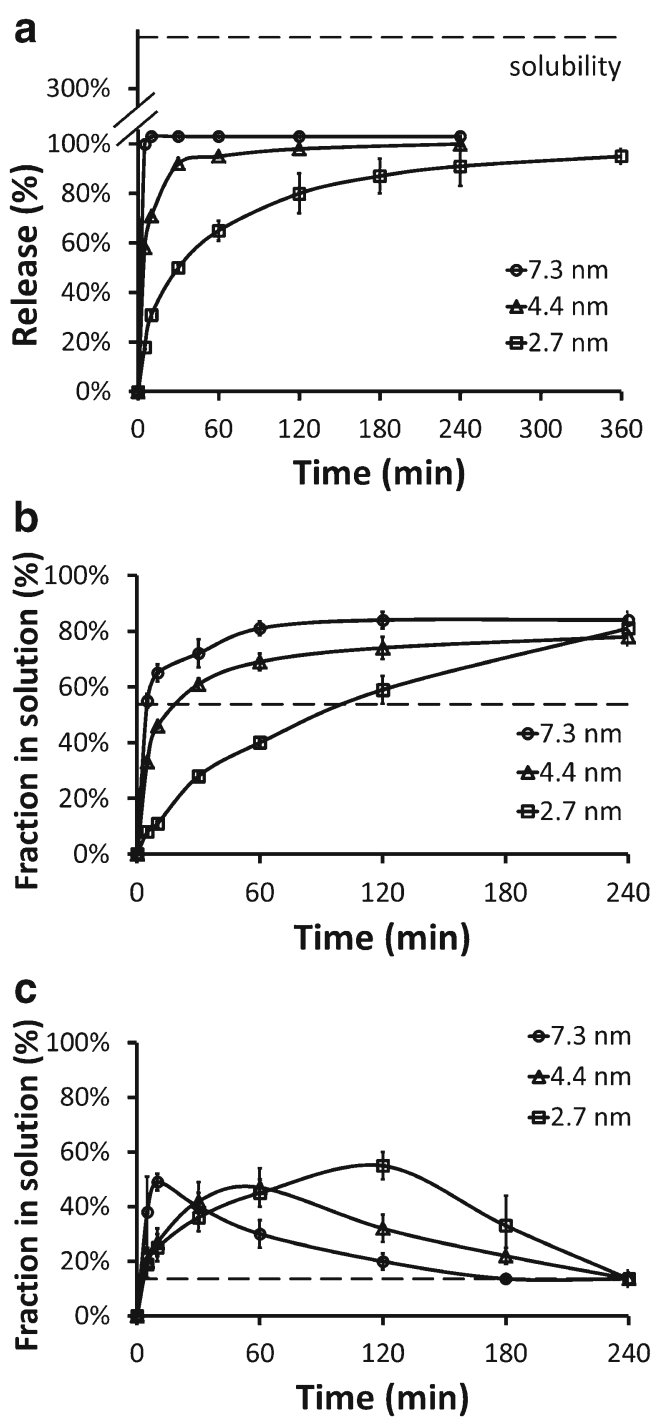

Fig. 2. In vitro dissolution profiles of amorphous fenofibrate from ordered mesoporous silica with varying pore sizes $(2.7,4.4$, and $7.3 \mathrm{~nm}$ ) under dissolution conditions with Sink Index $(S I)$ values of a $\mathrm{SI}=3.56$ (in FaSSIF+1\% SLS), b SI=0.538 (in FeSSIF), and c $\mathrm{SI}=$ 0.136 (in FaSSIF), calculated from Eq. 2. The dashed line represents the equilibrium solubility of fenofibrate in its respective dissolution medium. Figure adapted in part from Van Speybroeck et al. (18) (reproduced with permission from the European Journal of Pharmaceutical Sciences, Copyright Elsevier, 2010)

evidence on the effect of supersaturation generation rate (i.e., initial dissolution rate of supersaturating formulations), it is clear that exceedingly rapid dissolution of amorphous pharmaceuticals in the GI tract leads to fast precipitation manifested in a rapid decline in drug concentration, thereby leading to suboptimal oral bioavailability.

\section{SINK DISSOLUTION CONDITIONS: TO BE, OR NOT TO BE?}

The U.S. Food and Drug Administration's guidance on dissolution testing currently in force for immediate-release oral solid dosage forms dates back to August 1997 (22). In this guideline, the key objectives of dissolution testing include

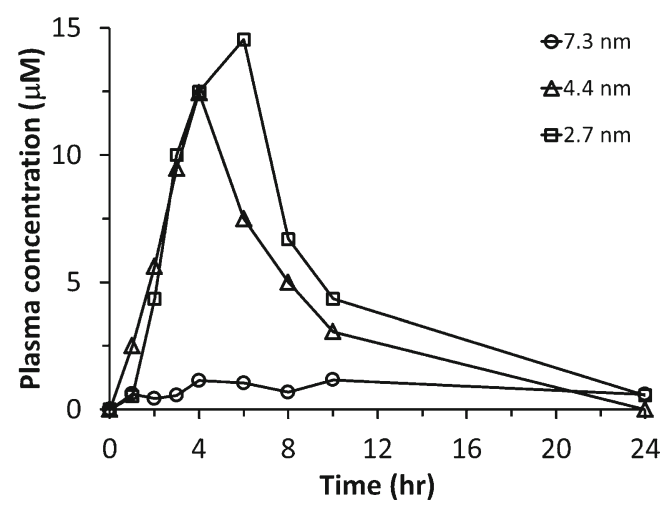

Fig. 3. In vivo PK profiles of fenofibric acid after oral administration of amorphous fenofibrate in ordered mesoporous silica with varying pore sizes $(2.7,4.4$, and $7.3 \mathrm{~nm})$ in rats under fasted conditions. Figure adapted in part from Van Speybroeck et al. (18) (reproduced with permission from the European Journal of Pharmaceutical Sciences, Copyright Elsevier, 2010)

the assessment of lot-to-lot consistency, confirmation of continuous product quality after certain changes (e.g., formulation, manufacturing process), and evaluation of new formulations. While the guideline clearly defines the apparatus, dissolution medium, hydrodynamics of agitation, and other aspects of dissolution methodology, it contains the statement that "sink conditions are desirable but not mandatory" (22), thus allowing flexibility in selecting the dissolution conditions. However, proposing a dissolution method under sink or nonsink conditions at times presents challenges to industry and the regulatory agency, especially for oral drug products containing supersaturating formulations. Current in vitro dissolution testing using compendial methodologies (i.e., United States Pharmacopeia (USP)) is conducted under perfect sink conditions, defined as the volume of dissolution medium at least three times that required in order to form a saturated solution of drug substance (23). It is quite evident

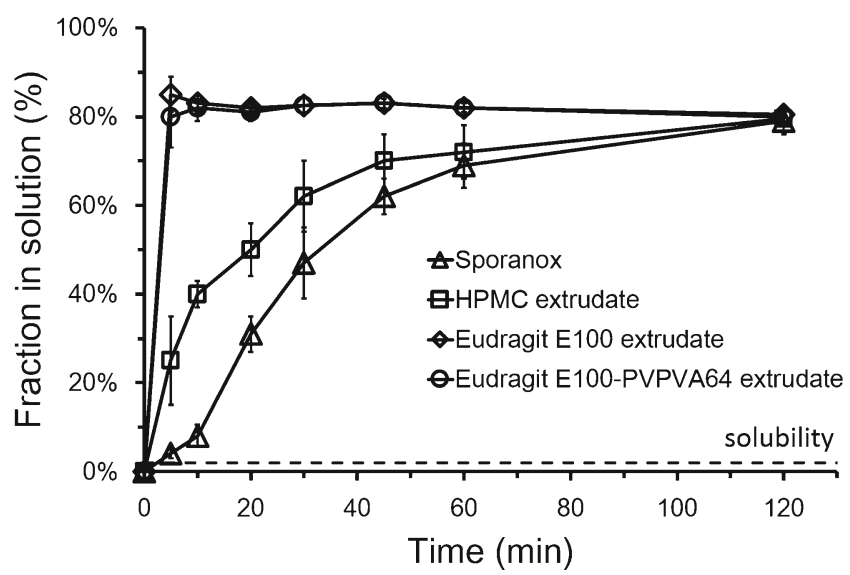

Fig. 4. In vitro dissolution profiles of Sporanox and other itraconazole ASD systems based on HPMC, Eudragit E100, and Eudragit E100-PVPVA64 prepared by hot-melt extrusion under dissolution conditions with Sink Index $(S I)$ value of 0.02 . The dashed line represents the equilibrium solubility of itraconazole (approximately $4 \mu \mathrm{g} / \mathrm{mL}$ ) at $\mathrm{pH} 1$ as described in the original publication. Figure adapted in part from Six et al. (20) (reproduced with permission from the European Journal of Pharmaceutical Sciences, Copyright Elsevier, 2005) 


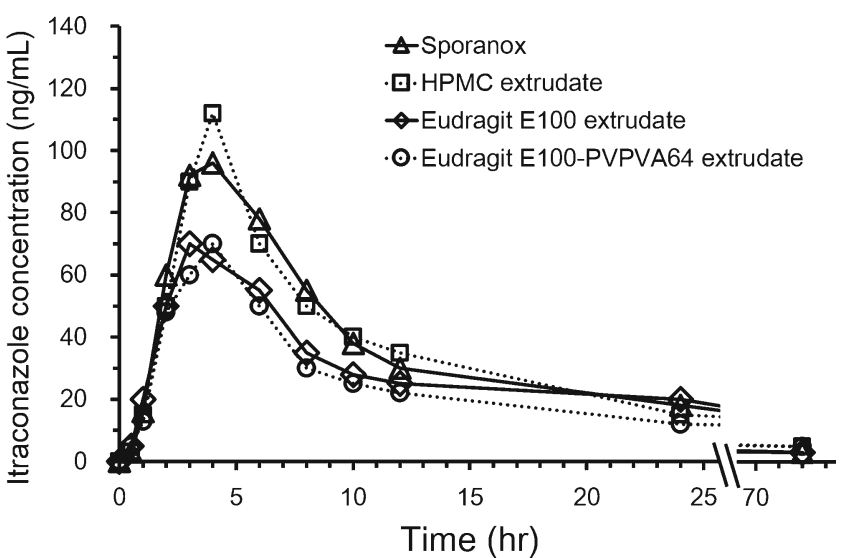

Fig. 5. Average plasma concentration-time profiles of itraconzole after oral administration of Sporanox and other itraconazole ASD systems based on HPMC, Eudragit E100, and Eudragit E100PVPVA64 in healthy human subjects $(n=8)$ with error bars omitted for clarity of trend. Figure adapted in part from Six et al. (20) (reproduced with permission from the European Journal of Pharmaceutical Sciences, Copyright Elsevier, 2005)

that this dissolution method would not be suitable for the direct assessment of enabling supersaturating formulations for the purpose of generating supersaturated drug solutions to improve the oral bioavailability of insoluble drugs. In addition, a physico-relevant dissolution testing involving solid-state analysis is recognized to be particularly important for predicting the in vivo performance (24). Following the advent of real-time and in situ analytical methods for dissolution testing (e.g., derivative UV spectroscopy), it is now possible to quantify drug concentrations in a supersaturated state without the interference of undissolved or newly formed submicron particles, thus allowing the generation of validated dissolution methods under nonsink conditions (25). Dissolution under nonsink conditions can build up drug supersaturation during the dissolution of supersaturating systems as commonly occurred under finite-volume conditions in the GI tract which can trigger the associated nucleation and crystallization events. As such, the application of nonsink conditions in supersaturation dissolution testing is generally recommended in order to evaluate the true performance of supersaturating formulations and to address the tendency for drug precipitation $(19,26)$.

In spite of the general consensus of endorsing nonsink conditions for supersaturation dissolution testing, there is no clear direction to develop dissolution methodology and to set dissolution specifications for supersaturating systems. In this case, one of the dilemmas may be attributed to the absence of a well-established definition of the degree of departure from perfect sink conditions on the sink-nonsink spectrum for dissolution testing of supersaturating formulations. To properly quantify such an extent of "nonsinkness," a dimensionless Sink Index (SI) has been introduced by Sun et al. (14) which is defined as follows:

$$
\mathrm{SI}=\frac{C_{\mathrm{S}}}{\text { Dose } / V}
$$

where $C_{\mathrm{S}}$ is the solubility of crystalline drug, $V$ the volume of dissolution medium, and Dose the total amount of drug in the test sample. Here, SI is really a ratio of the crystalline drug solubility to the drug concentration in the dissolution medium when the entire dose is dissolved. For supersaturating formulations, SI is also equivalent to the inverse of the maximum degree of supersaturation achievable. It is important to define SI for all dissolution experiments to better differentiate the sink/nonsink conditions since dissolution conditions corresponding to different ranges of SI values clearly affect the characteristic shape of dissolution profiles of supersaturating formulations as illustrated in Fig. 2, where the SI values are calculated from data presented by Van Speybroeck et al. (18) using Eq. 2. A dissolution method close to perfect sink conditions correlates to a large SI (e.g., SI $>3$ for the USP definition of sink conditions) (see Fig. 2a; equivalent to a maximum degree of supersaturation of $<0.33$ ). Consistent with the common practice of employing sink dissolution conditions for quality assurance, information gained here includes the total amount of released drug and the dissolution rate. Although a change in dissolution rate may be considered an indication of possible solid-state transformation in the sample, other characteristics such as surface properties (e.g., wettability, surface area) and burst effect may obscure such an analysis (24). When the dissolution testing is carried out under intermediate nonsink conditions with a SI value of around 0.5 for amorphous fenofibrate (see Fig. 2b; equivalent to a maximum degree of supersaturation of around 2), the corresponding dissolution profiles indicate that the formulations can indeed produce drug concentrations higher than the equilibrium solubility, thus confirming the ability of supersaturation generation. However, the absence of any decline in drug supersaturation in this case provides limited insights into the precipitation kinetics during the dissolution study because the relatively modest degree of supersaturation achieved here does not provide sufficient driving force for nucleation and crystallization to occur during the experimental timeframe. Under nonsink dissolution conditions with a small SI value of around 0.1 for amorphous fenofibrate (see Fig. 2c; equivalent to a maximum degree of supersaturation of around 10), the dissolution profiles follow the spring-and-parachute behavior reflecting the intricate interplay of dissolution and precipitation kinetics. In this case, the characteristic Cmax and AUC of these kinetic solubility profiles can be used to evaluate the extent and duration of supersaturation during in vitro dissolution. It should be noted here that although relative dissolution rates between formulations can still be observed in Fig. 2b, c, the total amount of released drug cannot be determined accurately since the fraction of drug dissolved in solution under nonsink conditions does not reach $100 \%$ due to the supersaturation and potential drug precipitation in the external bulk medium. It is also clear that the distinction between the intermediate nonsink conditions with SI value of around 0.5 and that of the nonsink conditions with small SI values of around 0.1 as depicted in Fig. $2 b$, c is directly related to the increase of fenofibrate solubility in the fed state over that in the fasted state (i.e., resulting in an increase of SI values in Fig. 2 b according to Eq. 2).

The use of complex solid-state formulations to improve oral bioavailability of poorly water-soluble drugs will inevitably lead to a multitude of dissolution behaviors, which in turn would require a well-defined dissolution methodology to best 
serve its purpose. To address the key question of how does one compare two nonsink dissolution conditions, the SI value discussed above can be conveniently used to differentiate dissolution methods for supersaturating formulations as it only requires knowledge on the equilibrium solubility (Cs) of the drug in physiologically relevant media (e.g., SGF, FaSSIF, FeSSIF), designated dose amount, and volume of the dissolution medium. Depending on the selected range of SI values (e.g., large, intermediate, or small) for the proposed dissolution method, pertinent information relating to the quality and performance of the test supersaturating drug products could be obtained by analyzing the characteristic trend of the resulting dissolution profiles as described above. Consequently, the dissolution specifications should be adjusted in accordance with the anticipated dissolution behaviors. For instance, single-point specifications may be appropriate to confirm the total drug content and dissolution rate for dissolution methods with high (e.g., Fig. 2a) and intermediate (e.g., Fig. 2b) SI values, respectively. However, for dissolution methods with small SI values which tend to magnify the supersaturation behavior (e.g., Fig. 2c), dissolution specifications would require a full kinetic solubility profile (i.e., multiple points) in order to reveal the underlying dynamics of drug dissolution and precipitation. Using the amorphous fenofibrate-mesoporous silica system with a pore size of $7.3 \mathrm{~nm}$ as an example, the dissolution specifications may be set as NLT (not less than) $95 \%(Q)$ in 10 min for a SI of 3.56 (Fig. 2a) and NLT $60 \%$ (Q) in 30 min for a SI of 0.538 (Fig. 2b) whereas multiple-point specifications including 40 $60 \%$ in $10 \mathrm{~min}, 30-50 \%$ in $30 \mathrm{~min}$, and $20-40 \%$ in $60 \mathrm{~min}$ may be needed for a SI of 0.136 (Fig. 2c). In addition, it is noteworthy that the extent of supersaturation generation and the crystallization propensity are all drug-dependent properties which will also affect the ranges of SI values where specific characteristic dissolution profiles may appear. Therefore, sink/nonsink conditions with a clearly defined SI value would be critical for defining an appropriate dissolution method to evaluate formulation performance and product quality of supersaturating formulations.

\section{DO NOT PUSH TOO FAR TOO FAST}

To fully characterize the dissolution profiles of supersaturating formulations under nonsink conditions with a small SI value, Sun and Lee have demonstrated the effects of supersaturation generation rate and initial degree of supersaturation on the overall kinetic solubility profiles $(27,28)$. They investigated these effects via an infusion experiment in which poorly water-soluble drugs (e.g., indomethacin) dissolved in a limited volume of water-miscible organic solvents (e.g., ethanol) were gradually infused into a dissolution medium. In addition, they proposed a model that predicts the kinetic solubility profiles from these infusion experiments, in which the observed time evolution of supersaturation (i.e., the rate of change of drug concentration) is expressed as the difference between dissolution and precipitation kinetics based on mass balance considerations. The rate of generation of supersaturation (i.e., dissolution kinetics) can be linear (e.g., infusion of drug solution with a fixed infusion rate) or nonlinear (e.g., dissolution of amorphous solids). The resulting time evolution of supersaturation and precipitation was predicted by a comprehensive mechanistic model based on the classical nucleation theory taking into account both the particle growth and ripening processes. In this case, the required molecular properties of the drug such as equilibrium solubility, crystal density, molecular diameter, diffusion coefficient in the dissolution medium, crystallization rate coefficient, and interfacial tension between drug solute and the dissolution medium were taken from literature data to simulate the precipitation kinetics. Both experimental data and theoretical results have revealed that a high rate of supersaturation generation (i.e., fast dissolution) (27) (see Fig. 6a) or a high initial degree of supersaturation $(28,29)$ (see Fig. 6b) inevitably causes an initial surge of supersaturation followed by a sharp decline in drug concentration due to rapid nucleation and crystallization events. However, a slower dissolution rate or a lower initial degree of supersaturation results in more gradual supersaturation
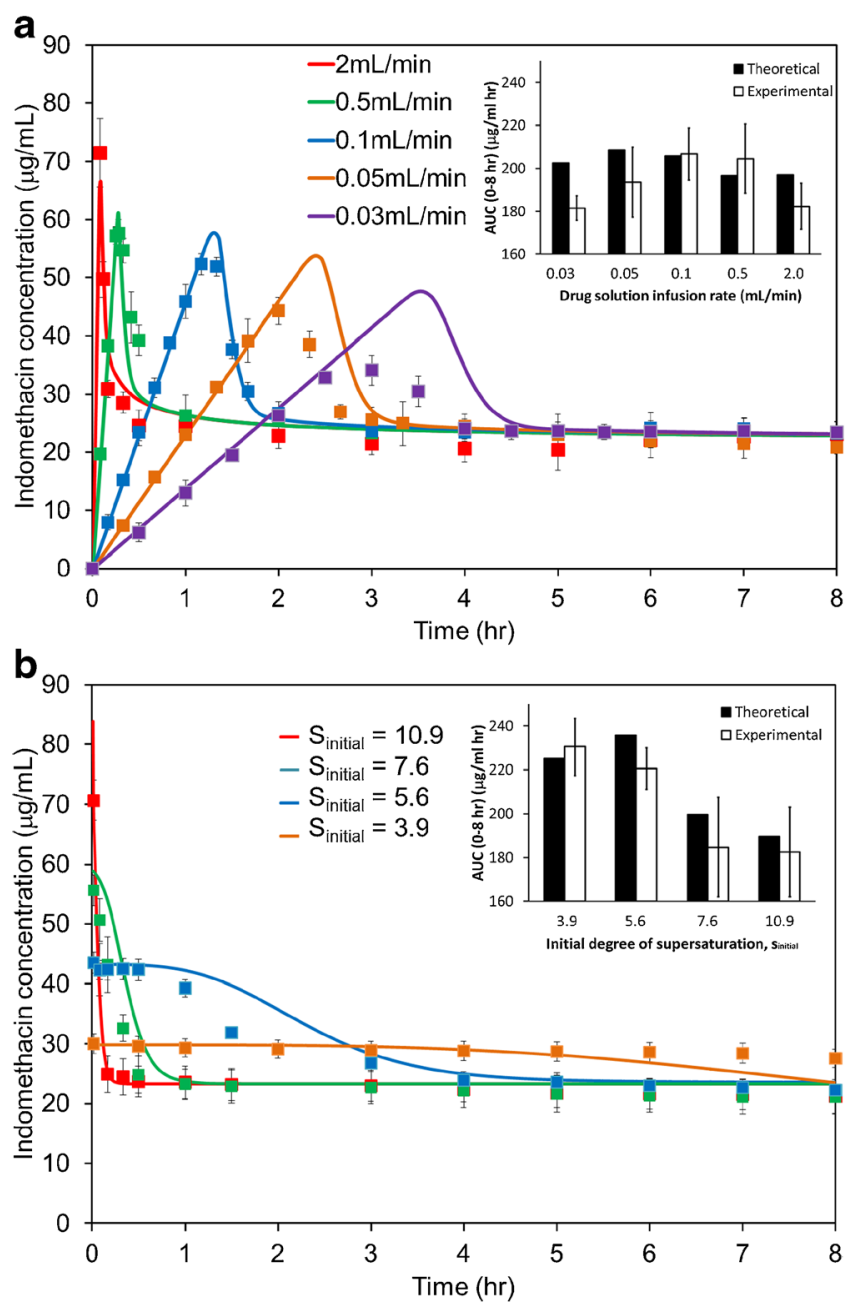

Fig. 6. Comparison of kinetic solubility profiles of indomethacin between experimental (symbols) and predicted (lines) data as functions of a supersaturation rate and $\mathbf{b}$ initial degree of supersaturation $\left(S_{\text {initial }}\right)$ generated from infusion of indomethacin solution in ethanol under nonsink dissolution conditions. Inset: Effects of drug infusion rate and initial degree of supersaturation on the AUC of the resulting kinetic solubility profiles. Figures adapted in part from Sun and Lee $(27,28)$ (reproduced with permission from Molecular Pharmaceutics, Copyright American Chemical Society 2013/2015) 
buildup followed by a slower de-supersaturation phase with diminished nucleation and crystallization rates. For the effect of supersaturation generation rate (Fig. 6a), the proposed model slightly underestimates the dissolution profiles at the highest infusion rate (i.e., $2.0 \mathrm{~mL} / \mathrm{min}$ ) and overestimates the dissolution profiles at lower rates (i.e., 0.05 and $0.03 \mathrm{~mL} / \mathrm{min}$ ) while the prediction at the intermediate infusion rates (i.e., 0.5 and $0.1 \mathrm{~mL} /$ $\min$ ) is quite good. For the effect of initial degree of supersaturation (Fig. 6b), the proposed model slightly overestimates all the dissolution profiles except for the lowest supersaturation level (i.e., $S_{\text {initial }}=3.9$ ). Overall, the critical trends of the predicted kinetic solubility profiles as a function of supersaturation generation rate and initial degree of supersaturation agree well with those of experimental data. Considering the fact that the mechanistic model simulation is based exclusively on physical parameters reported in the literature without employing any curve fitting in order to provide mechanistic insights into the resulting kinetic solubility profiles of ASD systems, the discrepancies mentioned above are therefore not surprising and the resulting predicted kinetic solubility profiles can be considered as quite good (please consult Ref. 27, 28 for detailed theoretical simulation of the effects of linear and nonlinear supersaturation generation rates).

To illustrate the associated solid-state transformation, the concentration-time curves presented in Fig. 6a, b can be converted to those of Fig. 7a, b for the characterization of precipitation kinetics under different infusion rates and initial degrees of supersaturation. It can be seen that the induction period of nucleation becomes shorter and the crystallization rate (slope of the rising portion) increases with the rate of supersaturation generation (see Fig. 7a) or the initial degree of supersaturation (see Fig. 7b). Similar trends of dissolutionprecipitation behavior have been observed by Kostewicz et al. in a transfer model for simulating the drug transfer out of the stomach into the intestine where the solution of a weakly basic drug in simulated gastric fluid is continuously pumped into a simulated intestinal fluid at different rates (30). The above lines of evidence suggest that an excessively high initial level of supersaturation or a very fast rate of supersaturation generation leads to a surge in maximum supersaturation followed by a sharp decline due to fast nucleation and crystallization; however, a sufficiently low degree of supersaturation or slow rate of supersaturation generation does not sufficiently raise the supersaturation level, resulting in a lower but broader maximum kinetic solubility profile.

The reported effects of rate and degree of supersaturation generation may in part explain the inverse relationship between the in vitro drug release rates and the extent of oral bioavailability as presented in Figs. 2 and 3. A rapid dissolution rate of amorphous fenofibrate in FaSSIF can generate a highly supersaturated drug solution within the first 30 min (Fig. 2c), which inevitably triggers the undesirable crystallization at the early stage of dissolution resulting in a smaller AUC of the kinetic solubility profile as seen in Fig. 2c. If similar uncontrolled nucleation and crystallization occur in the upper GI tract (i.e., in the gastric fluid), the precipitated solids will not be absorbed at the main absorption sites (i.e., duodenum and jejunum), thus significantly reducing the bioavailability when amorphous fenofibrate is orally administered to rats under fasted conditions as presented in Fig. 3. This supports the previous observation that fast dissolution of
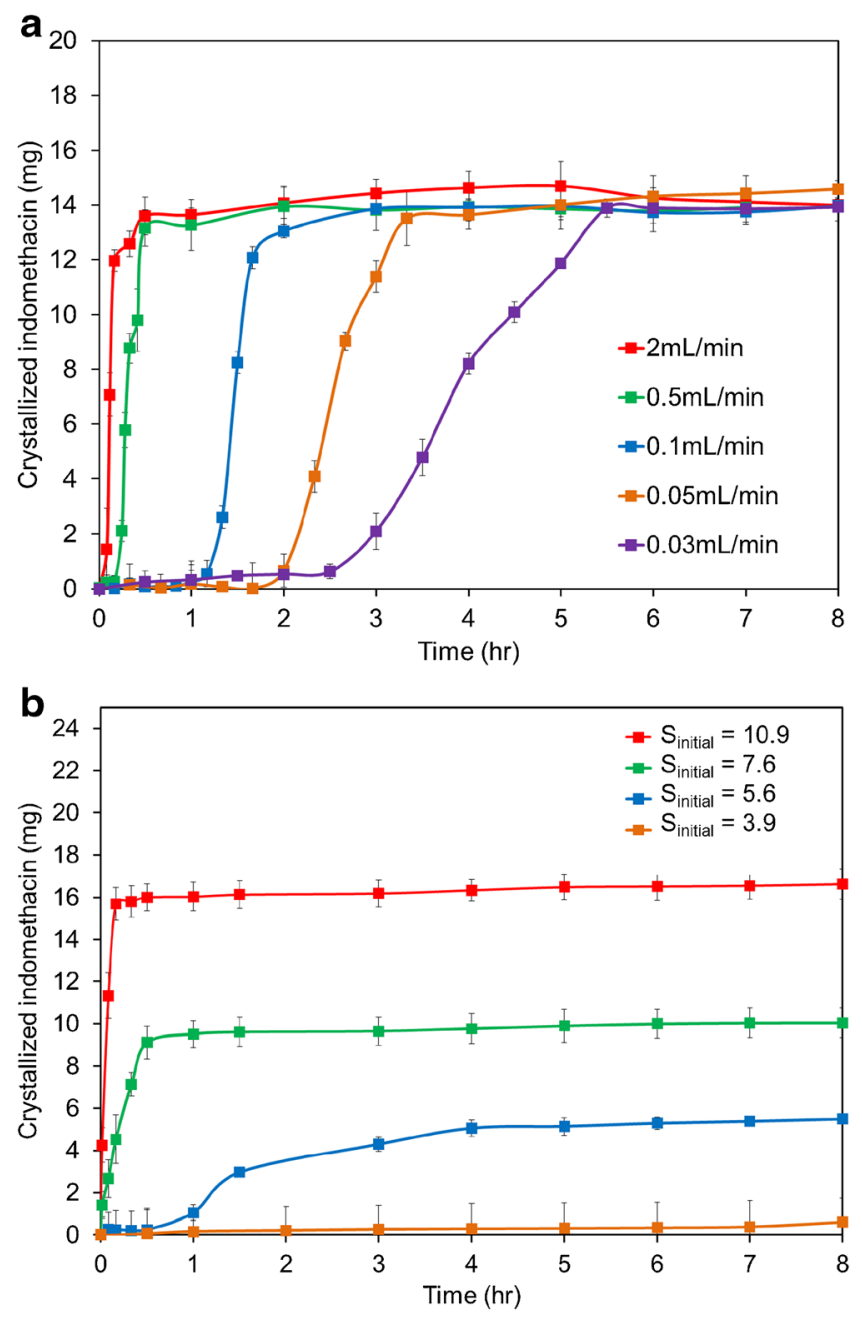

Fig. 7. Indomethacin crystallization kinetics as functions of a supersaturation rate generated with various drug solution (indomethacin in ethanol) infusion rates and $\mathbf{b}$ initial degree of supersaturation $\left(S_{\text {initial }}\right)$, converted from the kinetic solubility data described in Fig. 4. Figures adapted in part from Sun and Lee $(27,28)$ (reproduced with permission from Molecular Pharmaceutics, Copyright American Chemical Society 2013/2015)

supersaturating formulations does not always translate into an optimal in vivo performance (12). As shown in the insets of Fig. 6a, b, supersaturating formations with a modest dissolution rate or initial degree of supersaturation can generate a maximum exposure of dissolved drugs in the dissolution medium (i.e., maximum AUC) which are expected to prolong the supersaturated state. Whether this optimum AUC from the kinetic solubility profile translates to optimal oral absorption needs to be further established. Given the above evidence, if a poorly water-soluble drug formulated in supersaturating drug delivery systems is expected to exhibit in vivo dissolution/precipitation kinetics similar to the dissolution profiles presented in Fig. 2c, a gradual or intermediate rate of drug release from ASDs would be more desirable than a rapid "dose-dumping" which creates an instantaneous supersaturation followed by a sharp decline in drug concentration as a result of nucleation and crystallization (31). Hence, the effects of supersaturation generation rate and anticipated degree of supersaturation in vivo should be 
carefully considered in designing an optimal oral dosage form of supersaturating formulations in order to achieve an appropriate level of sustained solubility enhancement for poorly water-soluble drugs.

\section{THE "PHANTOM" SOLUBILITY OF AMORPHOUS PHARMACEUTICALS}

The effect of supersaturation generation rate also has direct implications on the prediction and measurement of solubility advantages of amorphous pharmaceuticals based on the generation of kinetic solubility profiles (27). In previous attempts of estimating the solubility advantages of amorphous solids, there usually exists a large discrepancy between the measured values and those predicted from various estimations of Gibbs free energy difference $(32,33)$. Prior to addressing the presented challenge, it is important to clarify the distinction between the equilibrium solubility (i.e., a thermodynamic property) and the non-equilibrium kinetic solubility (i.e., a kinetic property). The equilibrium solubility of a drug is defined as the maximum quantity of that drug which can be completely dissolved under given temperature, pressure, and solvent conditions (e.g., $\mathrm{pH}$ and chemical composition). The equilibrium solubility of a drug is determined from the drug concentration in a saturated solution in thermodynamic equilibrium with excess drug solids (i.e., crystalline). Similar to the equilibrium solubility, intrinsic solubility refers to the equilibrium solubility of an ionizable compound (e.g., an acid or base form) at a $\mathrm{pH}$ where it is fully un-ionized. On the other hand, the kinetic (metastable) solubility refers to the maximum achievable drug concentration in a supersaturated state (i.e., above the equilibrium solubility) and is typically determined from the maximum of a kinetic solubility profile.
Since a supersaturated solution is in a thermodynamically non-equilibrium state, phase transformation toward an equilibrium state is a thermodynamically favored process, kinetically driven by the free energy difference between the two states. In practice, the concentration range of the observed kinetic solubility profiles usually lies within an operating window between the equilibrium solubility and a threshold (or critical) supersaturation above which rapid uncontrolled precipitation tends to occur, commonly known as a metastable zone (MSZ) in the field of industrial crystallization $(34,35)$. The MSZ in a temperaturecomposition phase diagram is defined by the area between a binodal curve (i.e., a saturation point outside of which there is no driving force for nucleation) and a spinodal curve (i.e., a condition at which the metastable system becomes unstable, therefore representing the upper limit of MSZ and once crossed, spontaneous phase separation or spinodal decomposition must occur). In this region of the phase diagram, phase separation can take place and is controlled by a kinetic process described by the classical nucleation theory and crystal growth process. Improved oral bioavailability of supersaturating formulations is attributed to the kinetic solubility enhancement within the metastable zone width (MSZW) in a pharmacokinetically relevant timeframe. In this case, the MSZW depends on a number of intrinsic factors such as the drug's ability to generate supersaturation and its crystallization propensity. Within such a metastable zone, the time evolution of supersaturation is nevertheless a kinetic process in which a system inevitably moves from a nonequilibrium supersaturated state to an equilibrium saturated state by forming a separate solid phase. Since the observed kinetic solubility of non-equilibrium amorphous solids depends on the rate and the degree of supersaturation generation $(27,28)$, available experimental results highlight the underlying difficulty in determining a reproducible kinetic

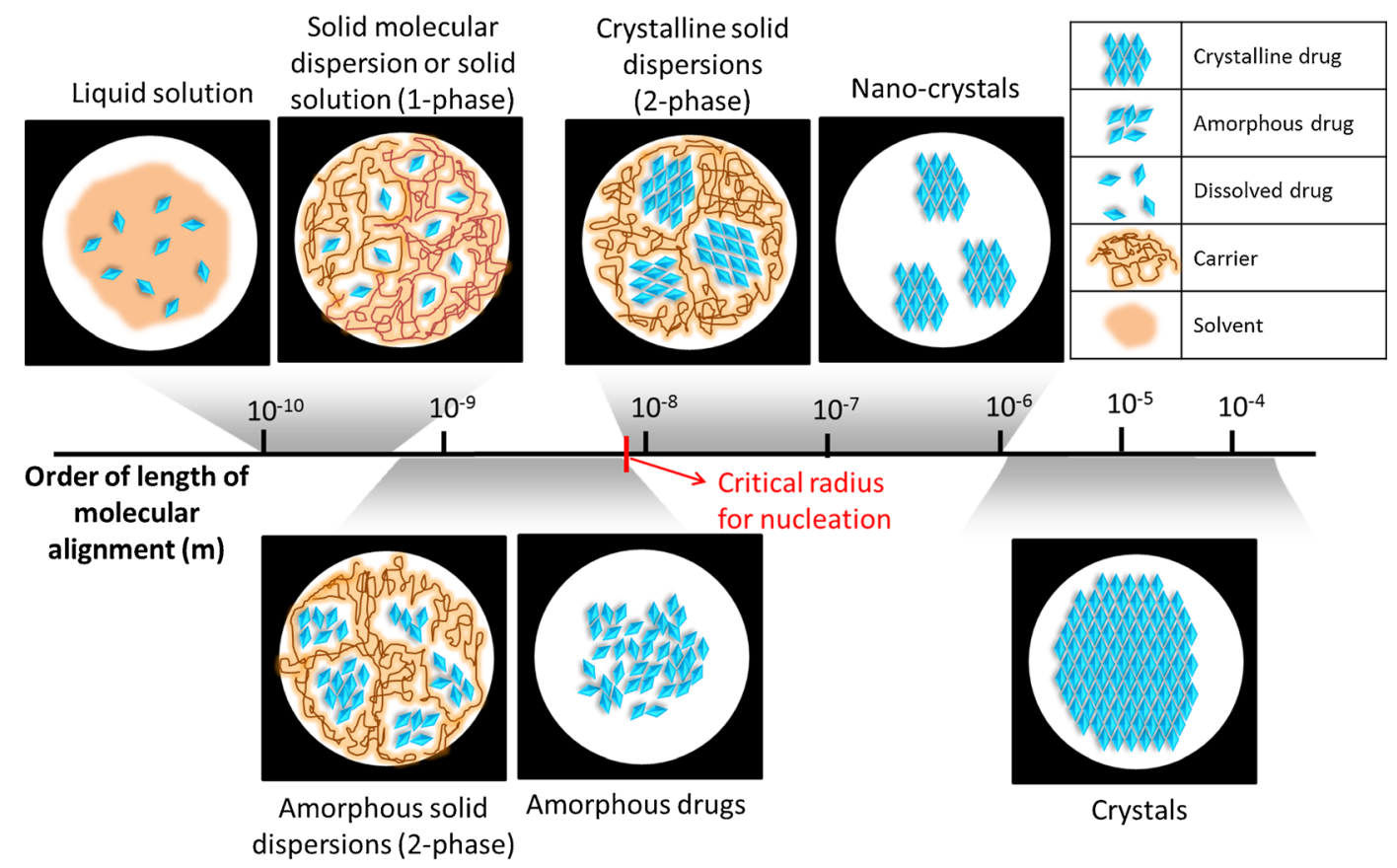

Fig. 8. Spectrum of molecular arrangements as a function of the order of length of molecular alignment 
solubility for amorphous pharmaceuticals. A theoretical framework has been proposed to account for the effects of rate and schedule of supersaturation on the MSZW calculation (34). Kinetically, when the supersaturating system moves away from equilibrium at a faster rate (i.e., higher rate of supersaturation generation in Fig. 7a) or to a greater extent (i.e., higher initial degree of supersaturation in Fig. 7b), a higher maximum supersaturation will be reached despite its ephemeral nature. The large discrepancy in the reported kinetic solubility values may well be the result of different rates of supersaturation generation in these different studies. Consequently, the true solubility advantage of amorphous pharmaceuticals cannot be accurately determined in practice.

From the viewpoint of solid-state chemistry, it is recognized that the molecular structure of amorphous solids lacks longrange crystalline order, rendering it similar to that of a liquid state. ASDs are usually a two-phase system containing a separate phase of amorphous solids with a short-range molecular order (i.e., length of only several drug molecules) compared to solid molecular dispersions (or solid solution) which is considered as a one-phase system in which individual molecules are molecularly dispersed in the carrier. The clustered molecules become stable new-phase nuclei for crystallization when the ordered molecular aggregate exceeds a critical radius (or size) for nucleation. Conceptually, the molecular arrangements of various solid-state pharmaceuticals can be expressed on a spectrum as a function of the characteristic order of length of molecular alignment as depicted in Fig. 8. It has been hypothesized that the solubility of a supercooled and liquidlike amorphous drug dissolved in an aqueous solution is analogous to that of two miscible liquids: Each component dissolves completely in the other regardless of the proportions in which two liquids are mixed (27). In fact, the molecular arrangement of one-phase solid solution appears to be more similar to that of a liquid solution than that of crystalline and amorphous solid dispersions (see Fig. 8). In most cases, pharmaceutical solid dispersion systems are present as a distribution of crystalline and amorphous solids in a carrier (i.e., a mixture or hybrid of several molecular structures shown in Fig. 8), rendering the experimental and theoretical analysis extremely complex. Furthermore, the observed kinetic solubility of non-equilibrium amorphous solids depends on the MSZW as well as the kinetic process of supersaturation generation as described earlier. Given the above considerations, rather than being attached to the idea of finding a fixed value of kinetically dependent solubility for amorphous pharmaceuticals, estimating the metastable zone width of poorly water-soluble drugs should be a better approach in determining whether or not these poorly soluble drugs are suitable to be formulated into ASDs.

\section{CONCLUSION}

Supersaturating formulations are a promising approach to improve oral absorption of poorly water-soluble drugs. Ideally, a supersaturated solution generated by the drug product in contact with the GI fluid should be maintained for a sufficiently long period to facilitate absorption before supersaturation-induced precipitation occurs. Pioneering studies have emphasized the importance of an elevated drug release rate from supersaturating formulations in dissolution testing under perfect sink conditions. Consequently, this early concept of enhanced dissolution rate has been, for quite some time, translated into formulation design strategies incorporating amorphous drugs into rapidly dissolving carriers. In the current article, we have attempted to summarize recent studies in order to bridge the gap in understanding the relationship between dissolution and precipitation kinetics of supersaturating formulations. Specifically, an in vitro-in vivo relationship identified from available data shows that an appropriate reduction in dissolution rate of ASD formulations could actually lead to improved oral bioavailability. In addition, both the rate and extent of supersaturation generation have been shown to have a profound impact on the overall evolution of supersaturation of amorphous pharmaceuticals over time. Exceedingly high rate and extent of supersaturation generation may be detrimental to the solubility enhancement and in vivo oral bioavailability of ASD formulations as shown by both in vitro and in vivo data as well as supported by results from modeling and simulation. In addition, physico-relevant dissolution methods need to be adequately defined with regards to the precise magnitude of departure from a perfect sink dissolution condition, for example using a dimensionless Sink Index (SI), in order to properly characterize and interpret the resulting nonsink dissolution profiles for product performance assessment and quality control purpose. Future research should focus on gaining fundamental understanding of the complex in vivo kinetics of dissolution, precipitation, and pharmacokinetic processes (e.g., absorption) of supersaturating formulations in the GI tract in order to benefit the development of supersaturating oral drug products.

\section{ACKNOWLEDGMENTS}

This work was supported in part by funding from the Natural Sciences and Engineering Research Council of Canada (RGPIN 06478-14). D. D. Sun was also supported by a University of Toronto Fellowship Award.

\section{REFERENCES}

1. Guzman HR, Tawa M, Zhang Z, Ratanabanangkoon P, Shaw P, Gardner CR, et al. Combined use of crystalline salt forms and precipitation inhibitors to improve oral absorption of celecoxib from solid oral formulations. J Pharm Sci. 2007;96(10):2686-702.

2. Warren DB, Benameur H, Porter CJ, Pouton CW. Using polymeric precipitation inhibitors to improve the absorption of poorly water-soluble drugs: a mechanistic basis for utility. J Drug Target. 2010;18(10):704-31.

3. Noyes AA, Whitney WR. The rate of solution of solid substances in their own solutions. J Am Chem Soc. 1897;19(12):930-4.

4. Nernst W. Theorie der Reaktionsgeschwindigkeit in heterogenen Systemen. Zeitschrift. 1904;47:52-5.

5. Goldberg AH, Gibaldi M, Kanig JL. Increasing dissolution rates and gastrointestinal absorption of drugs via solid solutions and eutectic mixtures II: experimental evaluation of a eutectic mixture: urea-acetaminophen system. J Pharm Sci. 1966;55(5):482-7.

6. Goldberg AH, Gibaldi M, Kanig JL. Increasing dissolution rates and gastrointestinal absorption of drugs via solid solutions and eutectic mixtures III: experimental evaluation of griseofulvinsuccinic acid solid solution. J Pharm Sci. 1966;55(5):487-92.

7. Simonelli A, Mehta S, Higuchi W. Dissolution rates of high energy polyvinylpyrrolidone (PVP)-sulfathiazole coprecipitates. J Pharm Sci. 1969;58(5):538-49. 
8. Simonelli A, Mehta S, Higuchi W. Dissolution rates of high energy sulfathiazole-povidone coprecipitates II: characterization of form of drug controlling its dissolution rate via solubility studies. J Pharm Sci. 1976;65(3):355-61.

9. Chiou WL, Riegelman S. Preparation and dissolution characteristics of several fast-release solid dispersions of griseofulvin. J Pharm Sci. 1969;58(12):1505-10.

10. Huang Y, Dai W-G. Fundamental aspects of solid dispersion technology for poorly soluble drugs. Acta Pharm Sin B. 2014;4(1):18-25.

11. Sun DD, Lee PI. Crosslinked hydrogels-a promising class of insoluble solid molecular dispersion carriers for enhancing the delivery of poorly soluble drugs. Acta Pharm Sin B. 2014;4(1):26-36.

12. Augustijns P, Brewster ME. Supersaturating drug delivery systems: fast is not necessarily good enough. J Pharm Sci. 2012;101(1):7-9.

13. Zahedi P, Lee PI. Solid molecular dispersions of poorly watersoluble drugs in poly (2-hydroxyethyl methacrylate) hydrogels. Eur J Pharm Biopharm. 2007;65(3):320-8.

14. Sun DD, Ju T-cR, Lee PI. Enhanced kinetic solubility profiles of indomethacin amorphous solid dispersions in poly (2hydroxyethyl methacrylate) hydrogels. Eur J Pharm Biopharm. 2012;81(1):149-58.

15. Sun DD, Lee PI. Probing the mechanisms of drug release from amorphous solid dispersions in medium-soluble and mediuminsoluble carriers. J Control Release. 2015;211:85-93.

16. Takahashi T, Yamaguchi M. Host-guest interactions between swelling clay minerals and poorly water-soluble drugs: II Solubilization of griseofulvin by complex formation with a swelling clay mineral. J Colloid Interface Sci. 1991;146(2):556-64

17. Hirasawa N, Ishise S, Miyata H, Danjo K. Physicochemical characterization and drug release studies of nilvadipine solid dispersions using water-insoluble polymer as a carrier. Drug Dev Ind Pharm. 2003;29(3):339-44.

18. Van Speybroeck M, Mellaerts R, Mols R, Thi TD, Martens JA, Van Humbeeck $\mathrm{J}$, et al. Enhanced absorption of the poorly soluble drug fenofibrate by tuning its release rate from ordered mesoporous silica. Eur J Pharm Sci. 2010;41(5):623-30.

19. Bevernage J, Brouwers J, Brewster ME, Augustijns P. Evaluation of gastrointestinal drug supersaturation and precipitation: strategies and issues. Int J Pharm. 2013;453(1):25-35.

20. Six K, Daems T, de Hoon J, Van Hecken A, Depre M, Bouche M-P, et al. Clinical study of solid dispersions of itraconazole prepared by hot-stage extrusion. Eur J Pharm Sci. 2005;24(2):179-86.

21. Six K, Verreck G, Peeters J, Brewster M, Van den Mooter G. Increased physical stability and improved dissolution properties of itraconazole, a class II drug, by solid dispersions that combine fastand slow-dissolving polymers. J Pharm Sci. 2004;93(1):124-31.

22. United States Department of Health and Human Services, Food and Drug Administration, Center of Drug Evaluation and Research. Guidance for industry: dissolution testing of immediate release solid oral dosage forms. August 1997.

23. United States Pharmacopeia and National Formulary (USP 38/ NF 33) <1092>The Dissolution procedure: development and validation May 1, 2015:1090-1097.

24. Aaltonen J, Rades T. Towards physicorelevant dissolution testing: the importance of solid-state analysis in dissolution. Dissolut Technol. 2009;16:47-54.

25. Liu P, De Wulf O, Laru J, Heikkilä T, van Veen B, Kiesvaara J, et al. Dissolution studies of poorly soluble drug nanosuspensions in non-sink conditions. AAPS PharmSciTech. 2013;14(2):748-56.

26. Brouwers J, Brewster ME, Augustijns P. Supersaturating drug delivery systems: the answer to solubility-limited oral bioavailability? J Pharm Sci. 2009;98(8):2549-72.

27. Sun DD, Lee PI. Evolution of supersaturation of amorphous pharmaceuticals: the effect of rate of supersaturation generation. Mol Pharm. 2013;10(11):4330-46.

28. Sun DD, Lee PI. Evolution of supersaturation of amorphous pharmaceuticals: nonlinear rate of supersaturation generation regulated by matrix diffusion. Mol Pharm. 2015;12(4):120315.

29. Patel DD, Anderson BD. Maintenance of supersaturation II: indomethacin crystal growth kinetics versus degree of supersaturation. J Pharm Sci. 2013;102(5):1544-53.

30. Kostewicz ES, Wunderlich M, Brauns U, Becker R, Bock T, Dressman JB. Predicting the precipitation of poorly soluble weak bases upon entry in the small intestine. J Pharm Pharmacol. 2004;56(1):43-51.

31. Gao P, Shi Y. Characterization of supersaturatable formulations for improved absorption of poorly soluble drugs. AAPS J. 2012;14(4):703-13.

32. Hancock BC, Parks M. What is the true solubility advantage for amorphous pharmaceuticals? Pharm Res. 2000;17(4):397404.

33. Alonzo DE, Gao Y, Zhou D, Mo H, Zhang GGZ, Taylor LS. Dissolution and precipitation behavior of amorphous solid dispersions. J Pharm Sci. 2011;100(8):3316-31.

34. Peters B. Supersaturation rates and schedules: nucleation kinetics from isothermal metastable zone widths. J Cryst Growth. 2011;317(1):79-83.

35. Kim K-J, Mersmann A. Estimation of metastable zone width in different nucleation processes. Chem Eng Sci. 2001;56(7):231524. 